\title{
SIMULATION IN EDUCATION AND TRAINING
}

\author{
J. Peter Kincaid \\ Ken K. Westerlund \\ Institute for Simulation and Training \\ 3100 Technology Parkway \\ University of Central Florida \\ Orlando, FL 32826 USA
}

\begin{abstract}
Historically, the use of simulation has been an important aspect of training in some fields (such as aviation). As the cost of computing power decreases simulation is now finding its way into training for other fields. As simulation moves into these other fields, it is increasingly moving away from traditional large hardware systems (e.g., full-motion simulators) to rich virtual environments such as serious games. However, matching the most efficient type and category of simulation to train specific learning needs is a specialized skill and there is a shortage or gap in the training of simulation specialists who can effectively design and employ training simulation. The skills needed by these professionals are presented and a program that has been established to train professionals in developing these required skills is discussed.
\end{abstract}

\section{INTRODUCTION}

Modeling and Simulation is breaking out from traditional areas of use (e.g., aviation and research) and emerging as an increasingly important tool for education and training. Part of the reason is that as the cost of computing power decreases it is becoming an economically viable media. However, economics alone do not fully account for the emergence of Modeling and Simulation's $(\mathrm{M} \& \mathrm{~S})$ growing incorporation in education (at all levels ranging from first grade through graduate education) and training. Why is the use of M\&S becoming so important? The following list provides a partial answer. Simulation:

- Is applicable to students of all levels and ages.

- Helps students see complex relationships that would otherwise involve expensive equipment or dangerous experiments.

- Allows for math, science, and technical skills to be taught in an applied, integrated manner.

- Provides students with new methods of problem solving.

- Provides realistic training and skills for a multitude of career areas. It is used extensively in science and industries.

- Is cost effective and reduces risks to humans.

The use of simulation in education does make a difference. Wenglinsky (1999) found that classroom simulation use was associated with academic achievement in math and also with many types of social improvements (e.g., motivation, class attendance, and lowered vandalism of school property). His study was based on National Assessment of Educational Progress (NAEP) scores for US students. Similarly, Kincaid, Donovan, and Pettitt (2003) found the use of simulation for training in the medical and military domains showed positive results.

\section{SIMULATION IN EDUCATION AND TRAINING}

\subsection{Historical Use of Simulation Training}

The application of simulation in education and training owes much to aviation training which has well over half a century of experience and "lessons learned" with simulators and simulation. Aviation simulators have long been used to train psychomotor tasks or sub-tasks and, within the past few decades, also tasks in the affective domain. Training in the affective domain has been targeted at increasing team performance and reducing human-error accidents. The results in aviation have been to practically eliminate air carrier accidents. For a long period of time the aviation field has recognized the training and safety 


\section{Kincaid and Westerlund}

benefits of using simulators. This recognition has manifested itself in the Federal Aviation Administration (FAA) pilot training in simulators to replace actual aircraft training (this reduces the cost of training), and many aviation insurance companies either require simulation training or offer reduced insurance premiums for companies that utilize simulator training. Additionally, the aviation field has benefited from a significant amount of research and guidance provided by the FAA to support and regulate the implementation of simulation in the training of pilots. The FAA publishes a number of documents, to include advisory circulars (ACs), which guide the employment of aviation training devices and simulators. For example, FAA-S8081-XX Airline Transport Pilot and Type Rating for Airplane and Helicopter Practical Test Standards includes a table (Task vs. Simulation Device Credit) that specifies the level of fidelity required by a device in order to receive credit for utilizing a given device. This table is supported by well defined tasks with specific conditions and standards. This level of guidance appears to be missing from simulation training as it is being applied to fields that have not traditionally employed simulation. For example, Kohn, Corrigan, and Donaldson (2000) hold the progress that aviation training made since World War II as a model for other fields and many companies are now developing more sophisticated simulators for training so that personnel in fields such as medicine can benefit from simulation.

\subsection{Medical Simulation Training}

The medical field is one of the fastest growing areas in modeling and simulation and has recently seen a proliferation in the use of training simulations and simulators. These simulators typically have hardware and software interfaces in order to provide training in the cognitive and motor skills required for various surgical procedures. For example, one company (Immersion Medical) produces an endoscopy simulator that allows trainees to practice three types of endoscopic procedures in an environment that is both safe for the patient, since a simulated patient is used, and provides task feedback to the trainee. According to Immersion Medical $\langle\mathrm{http}$ ://www. immersion. com/medical/ >: "Endoscopic procedures are some of the most commonly practiced medical procedures today." The device uses a robotic interface device and transmits force feedback to provide the trainee with tactile sensations (haptic technology) that mimic the feel of the actual procedure. Without these types of simulators it would be very difficult to train and assess the skills of the task being trained. According to Lian and Chen (2006) surgical training requires not only high fidelity in visual modeling, but it also in the area of haptic modeling. They go on to state that "a fundamental and typical application of surgical simulation, virtual suturing deals with technical problems such as soft tissue modeling, collision detection, and force modeling." Their device uses sophisticated mathematical equations to map surface textures for a more accurate virtual reality (VR) of human tissues and more precise haptic rendering of the human-VR interaction. They state that when "using this system, a user can feel the force change at different stage of the suturing process."

The government's website at <http://www.ahrq.gov/qual/advances/> lists a number of articles dealing with simulation and training. One such article, The Use of Surgical Simulators to Reduce Errors, draws some parallels with aviation training and NASA's system of aviation error reporting ("the NASA report"). According to the article: "The training of a surgeon includes the acquisition of a number of characteristics". These include a cognitive knowledge base, problem formulation and decision making abilities, appropriate psychosocial relationships, and other attributes that can be measured with objective testing, such as national board or specialty certifying examinations. Perhaps most critical to the surgeon, however, are the core technical skills of the profession. A battery of sophisticated devices is being created to teach and provide objective evaluations of the trainee's technical abilities. In medicine, just as in aviation, the range of simulators can run the spectrum from simple part-task trainers designed to teach a specific sub-task (e.g., the suture simulator) to more sophisticated simulators that can teach multiple tasks (e.g., the human patient simulator). These innovative state-of-the-art simulation devices can be very useful in teaching and evaluating medical student's cognitive and motor skills; however, without proper guidance or experience they can easily be misapplied, be over-or-under-utilized, and unnecessarily drive up training costs.

\subsection{Computer-based Games in Training and Education}

Computer-based games began as pure entertainment with simple "scoring" and human-computer interaction (e.g., Pong, Space Invaders, etc.). From that time computer-based games have developed at a very fast rate, a rate that has closely paralleled the development of more powerful computer hardware. As evidenced by products such as Halo 3, computer-based games have become very sophisticated and a large part of the entertainment market. These current state-of-the-art entertainment games use a wide range of technologies including: multi-player distributed game play, haptic feedback, mixed realities, and high fidelity audio and visuals. With the proliferation of computer-based games and simulations, they began to be incorporated in education and training as a strategy in order to achieve specific learning objectives.

As many of the purely entertainment games evolved some games began to take on more of an educational role, such as such as Reader Rabbit and Oregon Trail, which was first commercially available in 1985. Early education games designed strictly for the purpose of education and training focused on children. As these games started to take on a more "serious" as- 


\section{Kincaid and Westerlund}

pect, educators and researchers began to take note and look for ways to move them into a training/education role and for training complex tasks. One such game, Microsoft's popular Flight Simulator (MSFS) game, has very realistic navigation, terrain, and aircraft modeling that actually allows would-be pilots to plan and conduct relatively accurate flights. Vaden, et al. (1998) demonstrated that when an off-the-shelf game such as MSFS was combined with hardware that replicated the aircraft controls (e.g., functional throttle, yoke, rudder pedals, etc.) the game could have practical application in a learning/training environment. Gopher, et al. (1994) also tested the transfer of skills from MSFS to the flight performance of cadets in the Israeli Air Force flight school. They found the flight performance scores for two groups of cadets who received ten hours of training were much better in subsequent test flights than those with no game experience. According to Ruben (1999), computer-based games can provide the opportunity for knowledge or skills to be acquired and practiced in order to achieve an understanding of the underlying mental models of the subject being taught.

ActivChemistry, shown in Figure 1, is an example of an educational simulation of a chemistry lab. It is a chemistry construction kit that provides students with equipment and materials such as Bunsen burners, chemicals, and a wide variety of meters and gauges. Using these components, students perform experiments, gather and graph data, and learn about new concepts in interactive and dynamic lessons. ActivChemistry illustrates several advantages of the use of simulation as compared with real equipment. These advantages include safety (experiments can be done that would be too dangerous for most high school chemistry labs), economy (saves the cost of expensive equipment and materials), and learning efficiency (students using the program are not under the time pressures often found in standard chemistry lab periods and often complete exercises at a faster rate).

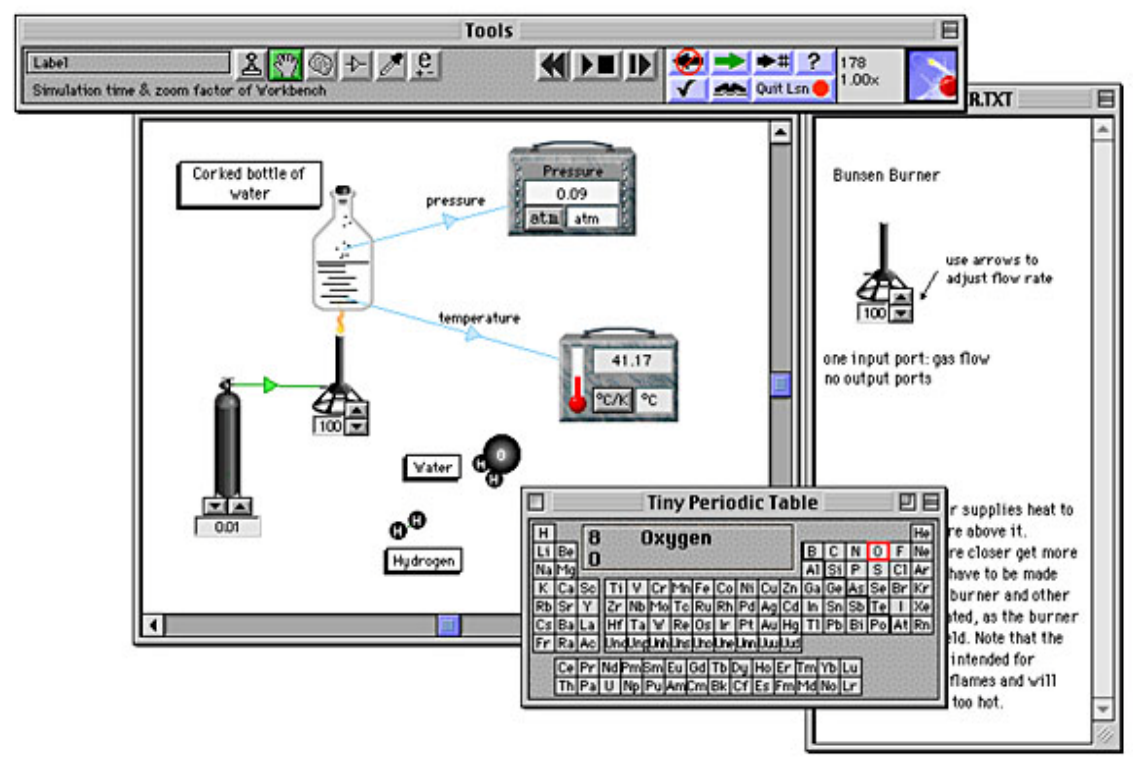

Figure 1: ActivChemistry. A virtual chemistry set construction kit grounded in chemistry theory

According to Shumucker (1999) simulations are very useful because they help students explore new concepts and gain an understanding of the interplay between related complex phenomena. Simulations typically incorporate free-play environments that provide the learner with experience in understanding how a set of conditions interact with each other. In the context of training and education "simulation is typically a software package that re-creates (simulates) a complex phenomena, environment, or experience." The learner is thus presented with the opportunity for some new level of understanding. PCbased simulations are typically interactive and grounded in some objective reality. Educational simulations are also usually based on some underlying computational model of the phenomena, environment, or experience and usually have some degree of unpredictability.

Simulation and games are examples of experiential instructional methods in that they are interactive and foster active learning. According to Brown (1999) both require a temporary suspension of disbelief as participants accept a false situation as temporarily real. Their differences lie in how players participate. In training simulations, players participate in situations or processes in order to learn about specific real-world settings or procedures. Recent studies have suggested that PC-based simulation games can produce a general transfer of cognitive skills that have application to a wide variety of domain-specific tasks. Other studies have used recent PC games for conducting psychological research on the cognitive processes involved in problem solving and strategy development (Gonzales and Cathcart 1995). 


\section{Kincaid and Westerlund}

\section{$2.4 \quad$ Serious Games}

Recent advances in PC technology such as high-speed processors, expanded memory, and high-performance video cards with 3D capability have made high quality synthetic environments inexpensive and highly immersive. This allows computer-based games to have a powerful engaging effect, which is one of their great strengths. Finding ways to engage students in training or educational games with the same intensity users engage with traditional games could revolutionize instruction. A number of organizations and researchers have recognized this and are involved with incorporating highly interactive computer-based games into a more serious role for training and instruction. These games are referred to as serious games. Two such organizations that would like to help facilitate the use and adoption of serious games are IITSEC and the Serious Games Initiative. The Serious Games Initiative (2007) website says: "The Serious Games Initiative is focused on uses of games in exploring management and leadership challenges facing the public sector. Part of its overall charter is to help forge productive links between the electronic game industry and projects involving the use of games in education, training, health, and public policy."

Tarr, Morris, and Singer (2002) examined the possibility of using PC simulation games for US military training. They concluded that PC games now have the capability to assist learning, transfer, and performance in a variety of domains, including substitution for real world training requirements. The PC gaming and simulation industry, largely driven by recent technology advances and consumer economics, has dramatically driven cost down while improving the quality and realism of games and desktop simulation technologies. Several different branches of the US military are exploring the possible use of serious games as a supplement to some aspects of training. The goal is to find low-cost training alternatives to train tasks that would normally require expensive equipment or involve hazardous conditions. One such serious game being developed by Camber Corporation is HuntIR Checkpoint Recon. The objective of this serious game is to train US Army Apache helicopter pilots and their training staff on cultural awareness issues and the rules of engagement (ROE) decision making process. Figure 2 depicts a screen capture from the game showing a simulated infrared image.

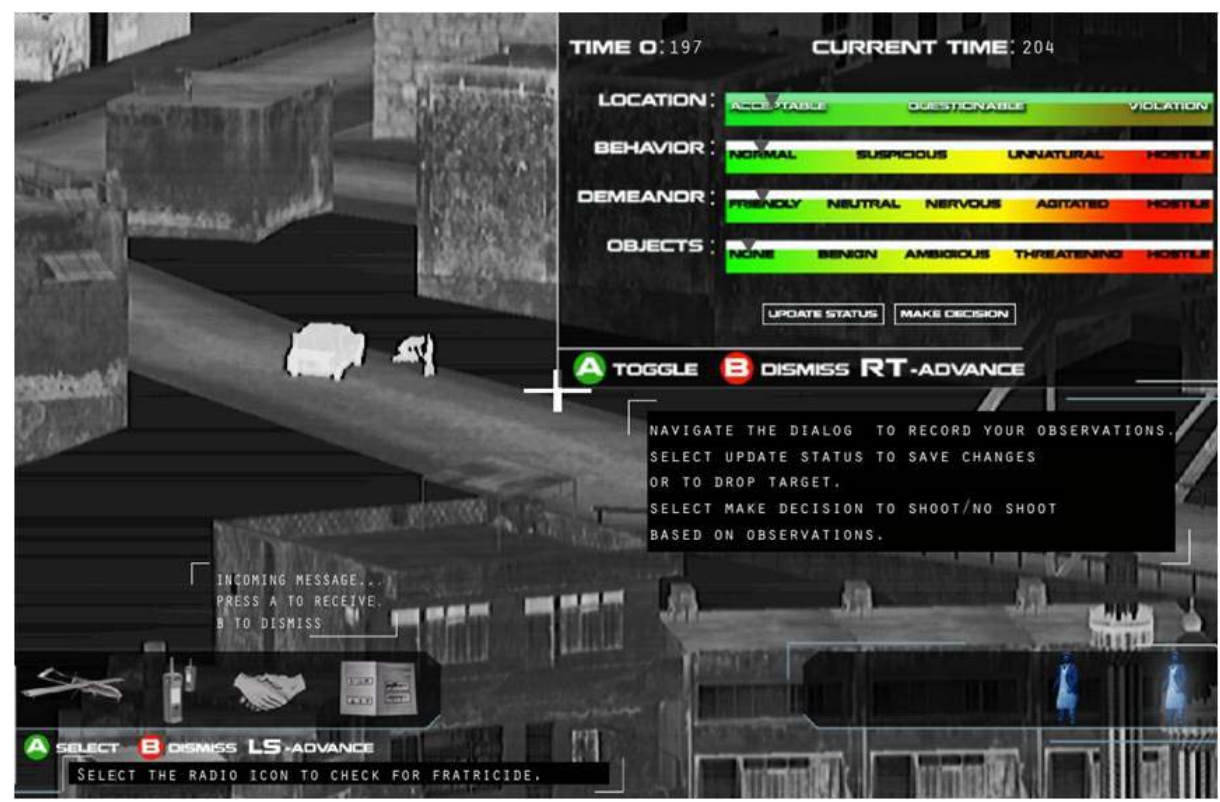

Figure 2: Screen capture of HuntIR Checkpoint Recon serious game

As with successful serious games it provides an immersive environment and is cognitively matched to the task to be trained. Since PC-based technology is at a point where human inclusion or immersion is fundamental, the capability and feasibility of applying PC games to enhance performance, training, and educational utility is evident. The question becomes how to select and employ specific games, or portions of games, to meet specific training requirements.

\subsection{Simulation and Instructional Theory}

Salas and Canon-Bowers (2000) conducted a review of the science of training that included a section on the use of simulation and games for training. The basic conclusion was that while simulations are widely used for training, how and why they work still needs further investigation. Additionally, developers often over-simulate or under-simulate for a given learning goal. A few studies have provided preliminary data (e.g., Jentsch and Bowers 1998); however, more systematic and rigorous evaluations of large-scale simulations and simulators are needed. Nonetheless, the use of simulation continues at a rapid pace in 


\section{Kincaid and Westerlund}

medicine (Kincaid et al. 2001), maintenance, law enforcement, and emergency management settings (Kincaid et al. 2003). Salas and his colleagues have noted that, unfortunately, simulation and simulators are being employed without much consideration of what has been learned about cognition, training design, or effectiveness. They concluded that there is a growing need to incorporate the recent advances in training research into simulation design and utilization.

Simulation devices vary in cost, fidelity, and functionality. Many simulation systems (including simulators and virtual environments) have the ability to mimic detailed terrain, equipment failures, motion, vibration, and visual cues. Other systems are less sophisticated and have less physical fidelity, but represent the knowledge, skills, and abilities (KSAs) to be trained (Jentsch and Bowers, 1998). In addition to the differences in the types of devices there are different categories of simulations. These include: live simulation, virtual simulations, and constructive simulations. Live simulations involve real people using real equipment (e.g., military training exercises using real equipment or role playing exercises). Virtual simulations involve real people operating physically and/or electronically simulated equipment in a simulated environment (e.g., full-motion flight simulators, surgical simulators, and video games). Constructive simulations involve simulated people using simulated equipment in a synthetic environment. These simulations make extensive use of mathematical and statistical techniques (e.g., the simulation of the output of an assembly line based upon various configuration changes). Selecting an inappropriate one can greatly increase training costs and/or, in some cases, cause negative learning transfer.

In order to maximize learning and return on investment (ROI) it is very important to select the proper category of simulation, to develop simulation-based training with the training objectives in mind, allow for the measurement of training process and outcomes, and incorporate formative evaluation of the training. This can best be accomplished by utilizing professionals skilled in the areas of $\mathrm{M} \& \mathrm{~S}$, instructional design, and human cognition.

\section{ACADEMIC PROGRAMS FOR M\&S}

"Where do simulation professional that can develop and apply the proper type of simulation to a training issue come from?" and "Exactly what skills do simulation training professionals need?" A number of academic institutions have begun to answer these questions by offering graduate programs in modeling and simulation. These programs are usually focused on the mathematical, hardware, or management aspects of M\&S. Recent analyses, presented at a workshop by Szererbicka, et al. (2000), concluded that there is an urgent national need for M\&S professionals. The workshop resulted in a strong consensus among individuals from industry, government, and academia that there is an exploding demand for M\&S professionals in all sectors of the economy. It also concluded that the majority of education and training offered in modeling and simulation lacks a firm pedagogical foundation. It was predicted that the lack of qualified graduates with solid education and training in M\&S would continue to get worse unless government and industry intervene and support rigorous M\&S education and training as a discipline. The workshop members concluded that graduate education in modeling and simulation needs to be considerably expanded to meet projected needs. Mostly MS\&T employers are forced to adopt an ad hoc solution to this national problem by hiring students from a variety of specialty areas (e.g., various disciplines of engineering, computer science, aviation training, and human factors) in the hope that capable students with these kinds of disciplinary backgrounds can be sufficiently well-trained on-the-job in the required M\&S skills. While such job-oriented training may be the only option for employers, it is unlikely to be the optimum way to produce the M\&S professionals needed to effectively develop and employ simulation targeted for education and training.

\subsection{The Education of a Simulation Professional}

Educational programs for training people who want to work in the modeling and simulation field are now being taught from the high school level through the Ph.D. level. One such graduate program at the University of Central Florida is attracting many students who have prior working experience in simulation, and who wish to receive formal training and to conduct research in simulation. Local industry leaders requested this program and have helped design the curriculum. They have expressed the desire that students have the capability to discover, design and develop simulation principles and methodologies, integrate simulation into decision processes of managers and leaders, and become professors of simulation programs in the country. Focus areas in the program include:

- Advanced Mathematical Modeling and High-performance Computing: advanced mathematical modeling and computing related to high-performance M\&S systems.

- Human Systems in M\&S: human modeling, situation awareness, decision-making, knowledge representation, intelligent architectures, human learning, human behavior, team training and performance, and human computer interaction.

- Interactive Simulation: requirements, design, development, and use of interactive simulation systems for knowledge transfer and training. 


\section{Kincaid and Westerlund}

- Networking and Computing Infrastructure: advanced, high-performance network test-beds, integrative architectures, and related technologies in support of distributed and large-scale simulation.

- Simulation Management: logistics, management, cost effectiveness analysis, and simulation-based acquisition and product development.

- Simulation Modeling and Analysis: simulation optimization, random phenomena, experimental design, environmental modeling, and simulation of biological phenomena.

- Computer Visualization in $M \& S$ : visual representation and computer graphics, including virtual environments, and aspects of computer graphics.

In addition to training on the more traditional hardware and software aspects of simulation, the UCF program provides the coursework electives required in order to prepare professionals to focus on M\&S for training and education.

\subsection{The Education of a Training Simulation Professional}

Table 1 shows a taxonomy of M\&S areas of knowledge for a number of disciplines and for various types of M\&S professionals (simulation model developer, scientist, and instructional simulation developer). The table is by no means comprehensive but is meant to show the breadth of knowledge and skills from various disciplines that are applicable to various $\mathrm{M} \& \mathrm{~S}$ professionals. The table also highlights the fact that the skills required by an instructional simulation developer, while similar in some respects, are significantly different than the skills/knowledge of other types of professionals in the M\&S field in other areas.

Table 1: A Discipline-Oriented Taxonomy of M\&S Professional Knowledge

\begin{tabular}{|c|c|c|c|c|}
\hline & Topic & $\begin{array}{c}\text { Simulation } \\
\text { Model Developer }\end{array}$ & $\begin{array}{c}\text { Scientist/ } \\
\text { Experimenter }\end{array}$ & $\begin{array}{l}\text { Instructional Simula- } \\
\text { tion Developer }\end{array}$ \\
\hline \multirow{6}{*}{$\begin{array}{l}\text { Math \& } \\
\text { Physics }\end{array}$} & Numerical Analysis & Low & High & Medium \\
\hline & Statistics & Medium & High & Medium \\
\hline & Linear Algebra & & Medium & \\
\hline & Differential Equations & Low & Medium & Low \\
\hline & Dynamics & & Low & \\
\hline & Electric Circuits & & Low & \\
\hline \multirow{4}{*}{$\begin{array}{c}\text { Industrial } \\
\text { Engineering }\end{array}$} & Programming & Low & High & Low \\
\hline & Nonlinear Optimization & Low & High & \\
\hline & Sensitivity Analysis & Low & High & \\
\hline & Cost Models & Medium & Low & Low \\
\hline \multirow{3}{*}{$\begin{array}{l}\text { Human } \\
\text { Factors }\end{array}$} & User Interface Design & High & High & High \\
\hline & Training Theory & Medium & High & High \\
\hline & Usability Testing & & & High \\
\hline \multirow{4}{*}{$\begin{array}{c}\text { Software } \\
\text { Engineering }\end{array}$} & Modular Program Design & High & High & \\
\hline & Verification \& Validation & High & & Medium \\
\hline & Testing & High & Low & Medium \\
\hline & Metrics & High & & \\
\hline \multirow{5}{*}{$\begin{array}{l}\text { Computer } \\
\text { Science }\end{array}$} & Database Systems & High & Medium & \\
\hline & Operating Systems & High & Medium & \\
\hline & Computer Networks & High & Low & \\
\hline & Distributed Systems & High & Low & Low \\
\hline & Artificial Intelligence & Medium & Low & \\
\hline \multirow{5}{*}{$\begin{array}{l}\text { Instructional } \\
\text { Technology }\end{array}$} & Instructional System Design & & & High \\
\hline & Media Selection & & & High \\
\hline & Task Analysis & Medium & & High \\
\hline & Instructional Evaluation & & & High \\
\hline & Learning Theory & & & High \\
\hline
\end{tabular}

When considering the employment of simulation Lewandowski (2007) advocates using "an engineering approach to attaining desired accomplishments from human performers by determining gaps in performance and designing cost-effective 


\section{Kincaid and Westerlund}

and efficient interventions." This engineering approach to employing simulation is similar to what has been occurring in the aviation training field and offers an opportunity to reduce human error accidents and increase training effectiveness. However, M\&S programs to develop these individuals need to be available in order to training the professionals with the ability to apply an "engineering approach" to the utilization of simulation in education and training. There is a serious shortage and corresponding demand for instructional simulation developers with specialized skills in the design and employment of simulation for efficiently meeting training needs. These simulation professionals need to be able to:

- Discover, design, and develop basic instructional simulation principles and methodologies.

- Design, develop, and manufacture simulation products for training.

- Manage and integrate simulation into training or human performance products.

- Teach other instructional simulation professionals.

\section{CONCLUSIONS AND RECOMMENDATIONS}

As we have seen, simulations can provide a safe environment that allows training and feedback on specific cognitive or psychomotor tasks far more frequently than would be the case if the trainee were waiting for actual equipment or specific conditions (e.g., medical simulators designed to train endoscopy and minimally invasive endovascular surgeries). Additionally, simulations provide an environment that is both safe for the patient, since a simulated patient is used, and provides task feedback to the trainee. We have also seen that simulations can also provide training and feedback on tasks in the affective domain to increase team skills, and reduce human error accidents. If one Boeing 747 crashes and kills 450 passengers it makes national headlines; however, if over 200 were to crash in a year (according to an Institute of Medicine report <http://www.ahrq.gov/qual/errorsix.htm> this is equal to the number of patients that die in hospitals each year as a result of medical human-error accidents) Americans would demand change. For a long period of time the aviation field has recognized the safety benefits of using simulator training. According to Kohn, Corrigan, and Donaldson (2000) the health industry "is a decade or more behind other high-risk industries in its attention to ensuring basic safety." They hold the progress that aviation training made since World War II as a model for the field of medical training and simulation could play a major role in training targeted at reducing these human-error accidents. However selecting the proper simulation is very important if the training is to be effective.

As training shifts from expensive high-end simulation solutions to serious games this problem will be magnified. Many of the games currently used for training seem to be simply showcasing new technologies or repurposing an entertainment game simulation rather than being targeted towards the elimination of an instructional gap. Well-defined instructional requirements for training and/or instructional games will need to be developed to ensure the games are accomplishing the goals for which they intended, and that they are not just simply providing entertainment. Technology continues to influence training systems, even though it is often employed without the benefit of findings from the science of training. For example, Vaden and Westerlund (1998) found that specifying the training requirements and integration strategies for games used in a training setting is still an area that needs further research. Most of the widely used simulation games have been developed for commercial reasons and including education features has typically come as an afterthought. Developing specialists with the specific skills to select the proper category of simulation and correctly apply it to the solution of a training problem should be a high priority of any M\&S training program (just as it is with hardware developers) and will result in more efficient training and improved safety. 


\section{Kincaid and Westerlund}

\section{REFERENCES}

Brown, A.H. 1999. Simulated classrooms and artificial students: The potential effects of new technologies on teacher education, Journal of Research on Computing in Education 32(2): 307-318.

Gonzales, F.E., and Cathcart, M. 1995. A procedure for studying strategy development in humans, Behavior Research Methods, Instruments, \& Computers 27(2): 224-228.

Gopher, D., Weil, M., and Bareket, I. 1994. Transfer of skill from a computer game trainer to flight, Human Factors 36(3): 387-405.

Jentsch, F., and Bowers, C. 1998. Evidence for the validity of PC-based simulations in studying aircrew coordination, International Journal of Aviation Psychology 8(3): 243-260.

Kincaid, J.P., Bala, S., Hamel, C., Sequeira, W.J., and Bellette, A. 2001. Effectiveness of Traditional vs. Web-based Instruction for Teaching an Instructional Module for Medics. IST-TR-01-06, Orlando: Institute for Simulation and Training, University of Central Florida.

Kincaid, J.P., Donovan, J., and Pettitt, B. 2003. Simulation techniques for training emergency response, International Journal of Emergency Management 1(3): 238-246.

Kohn, Linda T., Corrigan, Janet M., and Donaldson, Molla S., Editors. 2000. To Err is Human: Building a Safer Health System, Committee on Quality of Health Care in America, Institute of Medicine, National Academy Press, Washington, D.C. Available via <http://books.nap.edu/openbook.php?record_id=9728\&page=R1> [accessed October 11, 2007].

Lewandowski, William E. 2007. Success with Clinical Simulation = Assessment + Planning + Implementation. In Clinical Simulation, ed. Richard Kyle and W. Bosseau Murray, 471-478. New York: Academic Press.

Lian L. L. and Chen Y. H. 2006. Haptic Surgical Simulation: An Application to Virtual Suture, Computer-Aided Design \& Applications 3: 203-210.

Ruben, B.D. 1999. Simulations, games, and experience-based learning: The quest for a new paradigm for teaching and learning, Simulation \& Gaming 30(4): 498-505.

Salas, E., and Cannon-Bowers, J.A. 2000. The science of training: A decade of progress, Annual Review of Psychology 52: 471-499.

Tarr, R.W., Morris, C.S., and Singer, M.S. 2002. Low-Cost PC Gaming and Simulation: Doctrinal Survey, Army Research Institute Research Note, Alexandria, Virginia: Army Research Institute.

Serious Games Initiative. 2007. Serious Games Initiative. Available via <http:// seriousgames . org $>$ [accessed September 24, 2007].

Szezerbicka, H., Banks, J., Rogers, R.V., Oren, T.I., Sarjoughian, H.S., and Zeigler B.P. 2000. Conceptions of curriculum for simulation education, In Proceedings of the 2000 Winter Simulation Conference, eds. J. A. Joines, R. R. Barton, K. Kang, and P. A. Fishwick, 1635-1644. Piscataway, New Jersey: Institute of Electrical and Electronics Engineers, Inc.

Vaden, Eric A. \& Westerlund, Ken K. 1998. Microsoft Flight Simulator Training Study: Final Report (TSD-MSFS-98-1). Daytona Beach, FL.

Vaden, Eric A., Westerlund, Ken K., Koonce, Jefferson M., \& Lewandowski, William. 1998. The Use of Personal ComputerBased Aviation Training Devices in Ab Initio Flight Training, In Proceedings of the Human Factors and Ergonomics Society 42nd Annual Meeting: Chicago, IL.

Wenglinsky, H. 1999. Does it compute: The relationship between education technology and student achievement in mathematics. Princeton, New Jersey: Educational Testing Service.

\section{AUTHOR BIOGRAPHIES}

J. PETER KINCAID is a graduate research professor at the Institute for Simulation and Training, University of Central Florida. He directs the university's graduate programs in Modeling and Simulation and has been active in research related to simulation-based training for many years. He holds a $\mathrm{PhD}$ in human factors from the Ohio State University. His email is <pkincaideist.ucf.edu>.

KEN K. WESTERLUND is a PhD student in the University of Central Florida's (UCF) Modeling and Simulation (M\&S) program. He received Masters of Science degrees from Boise State University in Instructional and Human Performance Technology and from UCF in M\&S. He holds FAA Airline Transport Pilot and Flight Instructor certificates and has extensive experience in the utilization and application of simulation to training. His email is $<$ kwesterlund@CFL.rr.Com $>$. 